\title{
Alfred Werner 1866-1919, in memoriam
}

\author{
Von P. KarRer
}

\section{Dozentenschaft und Studentenschaft in den ersten Jahrzehnten chemischer Forschung in Zürich*}

Die Persönlichkeit Alfred Werners und seine Lehr- und Forschertätigkeit waren wesentlich von der allgemeinen Atmosphäre beeinflußt, die zu seinen Lebzeiten die beiden Hochschulen in Zürich umgab und in die Alfred Werner hineinwuchs. Es ist die Zeit etwa zwischen 1890 bis 1914. Der Geist jener Jahrzehnte wurzelte aber seinerseits in den Anschauungen, geistigen Strömungen und politischen Leidenschaften der vorausgegangenen Jahre.

Die Anfänge wissenschaftlicher Forschung in Zürich gehen auf die Gründung einer Theologenschule, das Carolinum, im Jahr 1525 durch UlRICH ZwINGLI zurück. In den darauffolgenden drei Jahrhunderten blieb dieses ohne bedeutende Änderungen bestehen. In jene Zeit fallen die ersten Anfänge naturwissenschaftlicher Forschertätigkeit durch Privatgelehrte. Ihr bedeutendster Vertreter war Conrad Gesner (1516-1565), der gleichzeitig Chorherr, Arzt, Zoologe, Mineraloge und Geologe war. Er schrieb eine Naturgeschichte des Tierreichs (Historia animalium), welche lange Zeit als das beste Werk über die damals bekannten Tiere galt, sowie die nicht minder wertvollen Opera botanica. - Auch Johann Jakob Scheuchzer (1672-1733) gehörte zu den großen Zürcher Naturforschern. Wie Gesner war er ein Enzyklopädist, zugleich Arzt, Naturforscher, Mathematiker und Philologe.

Während demnach die Anfänge botanischer, zoologischer, mineralogischer und ärztlicher Forschung in Zürich bis ins 16. und 17.Jahrhundert zurückreichen, fällt der Anfang der chemischen Forschung in unserer Stadt mit der Gründung der Universität im Jahre 1833 zusammen. Mit der Schaffung eines Lehrstuhls für Chemie wurde erstmals ein wissenschaftlich ausgewiesener Chemiker nach Zürich berufen.

* In dem vorliegenden Aufsatz über Alfred Werner habe ich Teile des Nekrologs, den ich nach Professor Werners Tod in den Helv. Chim. Acta veröffentlicht hatte, eingeflochten. Einige statistische und andere Angaben, die das Leben unserer Alma Mater Turicensis betreffen, sind der Festschrift zur Jahrhundertfeier der Universität Zürich Die Universität Zürich 1833-1933 und ihre Vorläufer entnommen. P. Karrer. 
Der erste Inhaber des Chemielehrstuhls an der Universität Zürich war KarL Löwig aus Kreuznach (Deutschland). Er war ein Schüler Gmelins, kam schon in jungen Jahren nach Zürich, verbrachte hier eine Assistentenzeit in der Meyerschen Apotheke zum Sternen an der Schiffände, habilitierte sich 1830 in Heidelberg und wurde drei Jahre später zum a.o. Professor für Chemie an die Universität Zürich gewählt. Obwohl er politisch nicht stark hervortrat, war er doch liberalen und freiheitlichen Ideen zugetan und nahm u. a. an der-erfolglos verlaufenen - Aktion gegen die Ausweisung Herweghs aus Zürich teil. Als Forscher und Lehrer hatte Löwig große Erfolge. Er arbeitete über organische Schwefelverbindungen, über natürliche Senföle und führte Analysen der Mineralwasser von Seewen, Schinznach und Baden aus.

Das chemische Laboratorium, in dem Löwig mit seinen Schülern tätig war, befand sich nach dem 1842 vollendeten Bau des heute noch bestehenden Kantonsschulgebäudes an der Rämistraße im Hochparterre dieses Gebäudes. Ein Bild dieses Laboratoriums mit den darin arbeitenden Dozenten und Chemiepraktikanden ist als Lithographie überliefert (Abb. 2). Es wurde erstmals von Emil Eidenbenz-Pestalozzi in der Professor Eduard Rüвel gewidmeten Festschrift (Verlag Schultheß, Zürich 1946) veröffentlicht und findet sich vorstehend reproduziert. Der Zeichner des Bildes ist nicht näher bekannt; nach E. Eidenbenz war es vielleicht Hartmann Krauer, der selbst auf dem Bild zu sehen ist (Nr. 4). Die Zeichnung ist nicht nur deswegen von Interesse, weil sie einen Einblick in das erste Zürcher Chemielaboratorium, das damals als sehr modern galt, erlaubt, sondern weil sie auch zeigt, wie sich die Zürcher Chemiestudierenden schon damals aus Angehörigen verschiedener Staaten zusammensetzten; neben neun Schweizern gehören der auf dem Bild sichtbaren Gruppe drei Deutsche und ein polnischer Graf an. Von mehreren dieser ausländischen Kommilitonen ist bekannt, daß sie freiheitlichen Ideen zugetan waren und sich auch für diese persönlich einsetzten.

Einige der auf dem Bild festgehaltenen Schüler Professor Löwigs sind später selbst erfolgreiche Forscher und Lehrer geworden. Nr. 2, Eduard Mathias Schweizer, war zuerst Lehrer an der oberen Industrieschule, 1841 habilitierte er sich, erst 23 jährig, an der Universität und wurde 1852 zum a.o. Professor ernannt. Er widmete sich besonders Gesteinsanalysen. Nr. 8 ist Hans Heinrich Landolt (1831-1910), der bedeutendste Schüler Löwigs. Nach der Vollendung des Studiums in Zürich ging er 1853 mit Löwig an die Universität Breslau, spezialisierte sich für physikalische Che- 
mie, zu deren eigentlichen Begründern er gehört, folgte später Berufungen an die Universität Bonn (1857), die Technische Hochschule Aachen (1869), die Landwirtschaftliche Hochschule Berlin (1880) und die Universität Berlin (1891). Seine Verdienste um die Forschung trugen ihm verschiedene Ehrungen ein; u.a. wurde er Mitglied der preußischen Akademie der Wissenschaften.

Der auf dem Bild als Nr. 5 bezeichnete Chemiepraktikant ist Friedrich von Beust (1817-1899), deutscher Staatsbürger. Nach 14 Jahren Militärdienst als Offizier trat er 1848 in die Redaktion der Kölnischen Zeitung ein, wandelte sich vom Monarchisten zu einem überzeugten Republikaner um, floh nach Paris und nahm an einem Aufstand in der Pfalz, später an einem solchen im Lande Baden teil. Die Flucht nach Zürich rettete ihn vor der Verhaftung. Er ließ sich nunmehr in dieser Stadt nieder und gründete hier die Beust-Schule in Hottingen (1854), die bald einen ausgezeichneten Ruf erlangte.

Nr. 4, Hartmann Krauer von Regensberg (Landwirt, dann Lehrer an der Töchterschule, Sekretär der Direktion für öffentliche Bauten und Privatdozent am Eidgenössischen Polytechnikum; Autorität im Weinbau). Nr. 6, Carl Cramers (1831-1901), wohnhaft am Zeltweg in Zürich, Sohn eines Müllers, studierte zuerst Chemie, dann unter dem Einfluß von Professor CARL Nägelı Botanik, habilitierte sich 1855 an der Universität Zürich, 1857 auch am Eidgenössischen Polytechnikum und wurde später zum Professor gewählt.

Die übrigen auf dem Bild dargestellten Chemiebeflissenen sind weniger bekannt geworden. Nr. 3 ist der Laboratoriumsabwart Scherrer, Nr. 7 Heinrich Zollinger, Apotheker in Hozgen (1826-1860). Nr. 9 David Nüscheler, Kaufmann in Zürich (1829-1881). Nr. 10 Heinrich LaNdis (1833-1915), Seidenfabrikant, Nationalrat. Nr. 11 unbekannt. Nr. 12 Johann Jakob Rahn (1830-1855). Nr. 13 CARL Heusler (1830-1862) von Basel, Chemiker. Nr. 14 Graf Alexander Sobansky von Piantkowka in Padolien. Sein Vater war polnischer Flüchtling und hatte sich in Pruntrut niedergelassen. - Nr. 15 RudoLF Роттноғ, deutscher Staatsangehöriger (wie Löwig aus Kreuznach).

Die Gründung der Universität Zürich im Jahre 1833 bedeutete für Stadt und Kanton Zürich den Beginn einer neuen geistigen Epoche. Nachdem am 22. November 1830, dem «Tag von Uster», die konservative Regierung durch eine liberal-demokratische gestürzt worden war, ergriff letztere die Initiative zur Reformierung des gesamten Schulwesens, dessen Spitze eine Universität bilden sollte. Bei den beschränkten finanziellen Mitteln, die 
dem Kanton zur Verfügung standen, bedeutete dies ein Wagnis; namentlich war nicht vorauszusehen, ob es gelingen würde, erstklassige Lehrkräfte für die neue kleine Hochschule zu gewinnen. Wenn sich diese Bedenken und Befürchtungen glücklicherweise als ungerechtfertigt erwiesen, so war dies hauptsächlich dem Umstand zu verdanken, daß in den ersten Jahrzehnten nach der Gründung der Zürcher Universität zahlreiche liberal denkende Dozenten aus dem benachbarten Ausland, wo reaktionäre Regierungen die Macht ausübten, in Zürich Asyl und Zuflucht suchten und glücklich waren, der Universität ihre Dienste zur Verfügung zu stellen. So hatte die Dozentenschaft der Universität Zürich bald ein internationales Gepräge.

Zu den in ihrer Heimat politisch verfolgten Dozenten, die darum eine Berufung an die Universität Zürich annahmen, gehörten z. B. der berühmte Mediziner Lucas Schönlein und der Naturphilosoph Laurenz Oken, welch letzterer der erste Rektor der Universität wurde; es gehörte dazu der vergleichende Anatom, talentvolle Dichter und Freiheitsstürmer Georg BüCHNER, der hervorragende Anatom Henle, der wegen Zugehörigkeit zu Burschenschaften in Deutschland gerichtlich verfolgt worden war. THEodor Mommsen, einer der vielseitigsten und bedeutendsten Gelehrten seiner Zeit, war in Leipzig wegen oppositioneller politischer Kundgebungen in eine schwierige Lage geraten und entlassen worden und konnte hierauf (1851) für die Universität Zürich verpflichtet werden. Auch Hermanv Köchly war wegen Beteiligung am Dresdener Aufstand vom Mai 1849 geflüchtet und wurde nun zum o. Professor der klassischen Philologie in Zürich ernannt. Weitere politische Flüchtlinge, die in den Lehrkörper der Universität Zürich aufgenommen wurden, waren E. OsenbrüGgen aus Dorpat und J.Temme aus Westfalen (beide juristische Fakultät). Johannes WisLicENUS, der dritte Inhaber des Lehrstuhls für allgemeine Chemie in Zürich, gehörte einer Familie an, die wegen politischen Verfolgungen aus Deutschland nach Amerika ausgewandert war. Auch die beiden sehr bekannten chemischen Technologen Bolley und EmIL Kopp, von denen ersterer von 1855-1870 und letzterer von 1870-1875 an der Е т H als chemische Technologen wirkten, waren aus politischen Gründen aus ihrer Heimat geflohen. Ersterer hatte an einer Aufstandsbewegung in Deutschland teilgenommen und war hierauf inhaftiert worden, letzterer kämpfte als Deputierter in Paris für freiheitliche Ideen und wurde 1849 in contumaciam verurteilt; er entzog sich der Strafe durch Flucht in die Schweiz. 
In den vierziger Jahren des vergangenen Jahrhunderts, besonders nach der Niederwerfung des badisch-pfälzischen Aufstands (1849) kamen zahlreiche geistig hochstehende Flüchtlinge in die Schweiz, insbesondere nach Zürich, von denen viele längere Zeit, manche auch dauernd, in ihrer neuen Wahlheimat blieben. Dadurch erhielt das geistige Leben Zürichs neue starke Impulse. $\mathrm{Zu}$ den prominentesten dieser ausländischen Gäste gehören z.B. Richard Wagner, Gottfried Semper, Georg Herwegh, Francesco de SANGTis u.a.m.

Die vielen ausländischen Gelehrten und Künstler, politische Flüchtlinge und auch andere, die in dieser Weise in den Bannkreis der Universität traten, bewirkten, daß sich an dieser jungen Hochschule Weltoffenheit, neue Ideen und freiheitliche Gedanken ungehindert entfalten konnten. Diese wirkten sich ungeschwächt in den kommenden Jahrzehnten aus. Auch Alfred Werner, dem diese Gedenkschrift gewidmet ist, fühlte sich von dem freiheitlichen Zürcher Geist angezogen. In seiner elsässischen Heimat, die damals zu Deutschland gehörte, aufgewachsen, innerlich sich aber als französischer oder zumindest elsässischer Bürger fühlend, begab er sich zum Studium an das Eidgenössische Polytechnikum nach Zürich. Er traf hier zahlreiche seiner engeren Landsleute, mit denen er regen Verkehr pflegte. In der Dozentenschaft der beiden Zürcher Hochschulen gab es viele Ausländer. Ein kosmopolitischer Zug wehte durch die Hörsäle dieser beiden Unterrichtsanstalten, in dem sich auch Alfred Werner wohlfühlte und der dazu beitrug, daß Zürich für ihn eine zweite Heimat wurde.

Der kosmopolitische Charakter der Zürcher Universität in den ersten Jahrzehnten ihres Bestehens und weiter bis zum Ersten Weltkrieg war aber nicht allein die Folge der Zusammensetzung der Dozentenschaft, sie wurde durch die Tatsache verstärkt, daß auch in der Studentenschaft das ausländische Element stark vertreten war.

In den ersten dreißig Jahren des Bestehens der Universität war der Prozentsatz ausländischer Kommilitonen noch relativ bescheiden, er schwankte zwischen 10 bis $15 \%$. Vom Jahr 1863 ab schnellte er aber unvermittelt auf $30 \%$, stieg 1873 auf $45 \%, 1893$ auf etwa $40 \%, 1903$ auf etwa $50 \%$ und hielt sich hierauf bis zum Ersten Weltkrieg auf einem Niveau zwischen 40 bis $50 \%$. Den größten Anteil in dem akademischen Völkergemisch stellten östliche Nationen, vor allem Russen und Polen, insbesondere solche jüdischer Religion, daneben aber auch Österreicher, Tschechen, Deutsche. Im Wintersemester 1868/69 wurde die erste Frau an der Universität Zürich immatrikuliert. Dies wirkte wie ein Funke, der eine gewaltige Invasion russischer, 
meist jüdischer Studentinnen an unserer Universität einleitete. 1869/70 waren von 14 immatrikulierten Studentinnen 7 Russinnen, 1872 von 63 immatrikulierten Frauen - hauptsächlich Medizinstudentinnen - 54 russischer Nationalität, im Jahr 1873 von 114 weiblichen Studierenden 100, die aus Rußland kamen.

Bei diesen russischen Emigrierten handelte es sich in überwiegendem Maß um politisch linksstehende, radikalisierte Jugendliche, Revolutionäre und Anarchisten, unversöhnliche Gegner des in Rußland damals bestehenden politischen Systems. Sie wurden in der Schweiz von erfahrenen Anarchisten, wie dem in Locarno niedergelassenen BaKunin, Rosa Luxemburg u.a., geleitet und weiter aufgehetzt.

Diese russischen Studentinnen waren schlecht vorgebildet und daher vielfach nicht fähig, ein erfolgreiches Studium zu bestreiten; dazu waren sie in politischer wie in sittlicher Beziehung oft zügellos. Im Zürcher Quartier latin, in der Gegend der Platte, hatte das Leben einen fremdländischen Charakter angenommen. Slawische und jüdische Elemente, alle arm, fast mittellos, beherrschten das Feld und ergaben sich revolutionärer Tätigkeit.

Es ist verständlich, daß sich in den Kreisen der schweizerischen Studenten Unzufriedenheit über das Überhandnehmen des ausländischen Elements bildete. Dieses nahm ihnen in Vorlesungen und Arbeitssälen die besten Plätze weg; die fremden Studenten und Studentinnen wurden auf Grund von ausländischen Zeugnissen immatrikuliert, die vielfach keinerlei Gewähr boten, daß diese Emigranten zum Studium reif waren; sie brachten eine politische Atmosphäre in die Universität, die einem seriösen Studium keineswegs förderlich war. Eingaben von einheimischen Studenten und Dozenten an die Erziehungsbehörden, die eine straffere Disziplin und strengere Immatrikulationsbedingungen für Ausländer forderten, wurde anfangs nicht entsprochen. Die Verhältnisse gestalteten sich indessen bald so unerfreulich, daß sich die Behörden schließlich gezwungen sahen, bei der Immatrikulation von Ausländern strengere Normen bezüglich des Alters (Minimalalter $18 \mathrm{Jahre}$ ), der Maturitätsausweise und der Sittenzeugnisse einzuführen. Dadurch gelang es, die unerfreulichsten Elemente aus der Studentenschaft zu eliminieren, diese bot aber immer noch, bis zum Ersten Weltkrieg, ein sehr buntes internationales Bild, in welchem der slawische Akzent nach wie vor stark vertreten war. Viele dieser dem Marxismus ergebenen Emigranten haben nach ihrer Rückkehr nach Rußland zweifellos ihr Teil zur Revolutionierung des Landes beigetragen. 


\section{Alfred Werners Jugend und erste Forschungsjahre}

Alfred Werner ist am 12. Dezember 1866 in Mülhausen (Elsaß) geboren. Seine Eltern lebten in bescheidenen Verhältnissen. Sein Vater war Fabrikinspektor und betrieb mit seiner Mutter Jeanne (geb. Teché) gleichzeitig noch ein kleines Bauerngewerbe. Vater und Mutter Werner waren nicht mehr jung, als Alfred zur Welt kam; dieser hatte mehrere Geschwister, die aber alle bis auf einen Bruder sehr früh starben. Die große Begabung Alfred Werners fiel inner- und außerhalb der Schule bald auf, und frühzeitig entwickelte sich bei ihm eine große Vorliebe für Chemie. In der Scheune des väterlichen Bauerngutes richtete er sich notdürftig ein chemisches Laboratorium ein; in dieser primitiven Arbeitsstätte machte er seine ersten Experimentalversuche. Da aber das Taschengeld knapp war, hat Alfred häufig anderen Leuten kleine Dienste getan, Holz gespalten usw., um einige Centimes zu verdienen, aus denen er sich dann Chemiebücher, Flaschen und Chemikalien erstand. Mit achtzehn Jahren pilgerte er mit der ersten, selbstverfaßten chemischen Arbeit zu Professor Noelting (in Mülhausen), um ihn um ein Urteil über das Manuskript zu ersuchen. Gleichzeitig richtete er an ihn die Frage, wie lange es ungefähr gehe, bis man Professor werde! Durch die ausweichende Antwort Noeltings ließ sich Alfred Werner in seiner Begeisterung für Chemie nicht irre machen. Als er ein Jahr später als Einjährig-Freiwilliger nach Karlsruhe übersiedeln mußte, benutzte er die Gelegenheit, um an der Technischen Hochschule mit dem Chemiestudium zu beginnen.

1886 zog Alfred Werner von Karlsruhe nach Zürich und setzte dort seine Studien am Eidgenössischen Polytechnikum fort. Hier wirkten damals als Vertreter der Chemie eine Reihe hervorragender Lehrer: Lunge, Hantzsch, Treadwell, welche die chemische Abteilung zu hohem Ansehen gebracht hatten und bei denen auch Werner eine ausgezeichnete Schule genoß. Über diese Studienjahre ist wenig überliefert; daß Alfred Werner schon damals eine gesellige Natur war, geht daraus hervor, daß er der Studentenverbindung «Stella» beitrat, die sich hauptsächlich aus Studierenden romanischer Zunge rekrutierte; hier konnte er wohl auch nähere Beziehungen zu seinen Landsleuten, den Elsässern, unterhalten. 1889 bestand er mit großem Erfolg - nur die Mathematik schien gewisse Schwierigkeiten zu machen - das Diplomexamen als technischer Chemiker und wurde hierauf Assistent bei Lunge. Gleichzeitig arbeitete er unter der Anleitung von Hantzsch an der Dissertationsschrift: Über die räumliche Anordnung der Atome in stickstoffhaltigen Verbindungen, die in ihrer Bedeutung weit über den gewöhnlichen 
Rahmen einer Dissertation hinausreicht und die scharfe Beobachtungsgabe und das selbständige Denken des jungen Gelehrten gegenüber Überliefertem enthüllte. Nach der Promotion ging Werner für ein Semester ins Collège de France nach Paris, um sich unter Berthelots Leitung weiterzubilden.

Wieder nach Zürich zurückgekehrt, habilitierte er sich 1892 am Polytechnikum mit der Schrift: Beiträge zur Theorie der Affinität und Valenz. In dieser finden wir, wenn auch zum Teil noch skizzenhaft, schon Gedanken, die wir heute zum köstlichsten Gut Wernerschen Schaffens zählen. Die ein Jahr später erschienene Arbeit: Beiträge zur Konstitution anorganischer Verbindungen zeigt Werner bereits auf einer solchen Höhe chemischen Denkens und Gestaltens, daß seine Fachgenossen Mühe hatten, seinen bahnbrechenden Ideen zu folgen. Losgelöst von überlieferten Anschauungen ging er hier daran, die experimentellen Resultate anorganischer Forschung nach neuen Gesichtspunkten zu erklären.

Diese ausgezeichneten Arbeiten veranlaßten den Zürcher Regierungsrat, als Nachfolger für den 1893 vom Lehrstuhl für Chemie an der Universität zurücktretenden Victor Merz in erster Linie Alfred Werner in Aussicht zu nehmen. Erst $27 \mathrm{j}$ ährig, wurde dieser zum Extraordinarius an der Universität gewählt und zwei Jahre später zum Ordinarius befördert. Die Wahl vollzog sich allerdings nicht ohne Aufregungen. Wie es oft so geht, wurde auch Werner ein anderer Kandidat gegenübergestellt, und das Wahlgeschäft nahm solche Formen an, daß es Werner vorzog, in den Zermatter Bergen den Ausgang der Wahl abzuwarten. Seine Freude war groß, als diese dann auf ihn fiel. Damit erschloß sich ihm eine große und dankbare Lebensaufgabe. Die ersten Jahre seiner Tätigkeit als Hochschullehrer waren allerdings nicht leicht. Schon der Umstand, daß zwischen ihm, den Assistenten und Studierenden ein nur geringfügiger Altersunterschied bestand, erforderte von seiner Seite viel Takt. Dazu kam, daß Werner, wie dies nicht anders sein konnte, noch wenig Laboratoriumserfahrung zu Gebote stand. In der Erkenntnis, daß er nicht nur Anregungen zu geben hatte, sondern auch selbst noch solcher bedurfte, richtete er daher Diskussionsabende ein, an denen er sich mit anderen Dozenten und Schülern über chemische Fragen aussprechen konnte.

Diese ersten Universitätsjahre lassen uns aber noch in anderer Beziehung einen Blick in sein Geistesleben werfen. In seinen Arbeiten aus jener Zeit finden sich organische und anorganische in buntem Wechsel neben- und durcheinander. Man hat den Eindruck, daß Werner damals mit sich selber noch nicht ganz einig war, ob er sich die eine oder andere Richtung als 
Lebensaufgabe wählen sollte. Er schwankte. Es war jene Zeit, in der die organische Chemie in einer einzigartigen, stürmischen Entwicklung begriffen war und durch ihre glänzenden Erfolge die anderen Zweige unserer Wissenschaft in den Schatten drängte. Auch Werner war durch ihren Glanz geblendet. Ihn zog es zwar innerlich mehr zur anorganischen Forschung, die aber damals kaum solche Erfolge versprach und bei weitem nicht so allgemein interessierte wie die organische Richtung. Mit seinem damaligen Kollegen Privatdozent Dr. Aug. Bischler hat er sich öfter über diesen Zweifel unterhalten, und es ist ein glückliches Geschick, daß Dr. Bischler diese Zweifel zu zerstreuen suchte und ihm zu dem riet, zu dem es ihn innerlich trieb, zur anorganischen Forschung. So konzentrierte sich Werner mehr und mehr auf sein eigentliches Lebenswerk, die theoretische und experimentelle Bearbeitung anorganischer Verbindungen höherer Ordnung. Bis gegen das Jahr 1900 hin finden wir unter seinen Veröffentlichungen noch solche organischen Inhalts, dann brechen sie ab. Von da an widmet er seine ganze Arbeitskraft der konsequenten Verfolgung und dem Ausbau der Koordinationstheorie.

Ins Jahr 1895 fällt Werners Verheiratung mit EmmA GIEsker von Zürich. Aus der glücklichen Ehe entsprossen ein Sohn (Alfred) und eine Tochter (Charlotte), an denen der Vater mit großer Liebe hing. Wir freuen uns, daß die Tochter, Fräulein Charlotte Werner, die Ehrung miterleben kann, welche die Chemiker der ganzen Welt anläßlich des 100. Geburtstages ihres berühmten Vaters diesem heute bereiten. Gleichzeitig mit seiner Verheiratung ließ sich Werner ins Schweizer Bürgerrecht aufnehmen. Er, der als französischer Bürger geboren wurde, bewahrte seinem Vaterland auch nach der Einverleibung des Elsaß an Deutschland seine Sympathie und Liebe und fühlte sich dem französischen Kulturkreis verhaftet, wenn er auch der deutschen Wissenschaft die größte Hochachtung entgegenbrachte und freundschaftliche Beziehungen mit verschiedenen deutschen Kollegen unterhielt. Seine ausgesprochen demokratische Denkweise erleichterte ihm das Hineinwachsen in schweizerische Verhältnisse. Seit seiner ersten Berührung mit unserem Land im Jahre 1886 fühlte er sich in diesem wohl. Der Besuch des Eidgenössischen Polytechnikums dürfte seine Zuneigung zu unserem Staat weiter gefördert haben. Er hat sich in der Folgezeit in Zürich vollkommen eingelebt und hier eine wirkliche zweite Heimat gefunden. Den Zürcher Dialekt sprach er so gut, daß niemand auf den Gedanken kam, seine Wiege sei im Ausland gestanden. - Mit Politik hat sich Alfred Werner nicht beschäftigt, aber er nahm regen Anteil an allem, was das öffentliche 
Leben berührte, und er brachte besonders auch allen Schulfragen reges Interesse entgegen. Als Mitglied der Aufsichtskommission des kantonalen Gymnasiums hatte er dazu willkommene Gelegenheit.

\section{Das Chemische Universitätsinstitut Zürich}

Als Alfred Werner das Extraordinariat für Chemie an der Universität antrat, war der chemische Unterricht der Universität im alten Chemiegebäude an der Rämistraße hinter dem Polytechnikum untergebracht. Dieses Gebäude, das bis zum Jahre 1887 als Chemie-Institut des Polytechnikums gedient hatte, war - auch nach damaligen Begriffen - äußerst primitiv und unzulänglich. Neben der Chemie waren in ihm noch die Chemie der Kantonsschule, der Kantonschemiker, die Hygiene und die Pharmakologie untergebracht. Die steigende Schüler- und Praktikantenzahl wurde von den zur Verfügung stehenden Räumlichkeiten kaum gefaßt. So war Professor Werner gezwungen, unter sehr ungünstigen äußeren Umständen seine wissenschaftlichen Arbeiten auszuführen. Nur die analytische Abteilung war in Räumen eingerichtet, die man heute noch vielleicht mit dem Namen Laboratorium belegen würde, die präparativen Arbeiten und die Doktoranden waren auf roh ausgebaute ehemalige Keller und Holzbehälter verwiesen, in denen auch um die Mittagsstunde künstliche Beleuchtung notwendig war. Titrationen konnte man daher hier nicht ausführen. Der kalte, zementierte Kellerboden, die schlechte Heizung und die Unmöglichkeit einer richtigen Ventilation machten diese Räume in hohem Grade ungesund. Sie verdienten den Namen Katakomben, den die Studierenden ihnen beigelegt hatten, mit vollem Recht (Abb. 3).

Auch die apparative Ausstattung dieser Arbeitsräume war außerordentlich bescheiden, um nicht zu sagen primitiv. Die geringen Institutskredite erlaubten die Anschaffung selbst einfacher Apparaturen, wie z.B. Autoklaven, optische Meßinstrumente usw., nicht. Als im Jahre 1833 die Zürcher Regierung im Anschluß an die Gründung der Universität beschloß, auch der Chemie ein Heim zu bieten, wurden zur Anschaffung von medizinischen und chirurgischen Apparaten, Instrumenten für Physik und Chemie usw. und zur Errichtung eines chemischen Laboratoriums insgesamt 3200 Schweizer Franken bewilligt sowie auf die Dauer von sechs Jahren ein nachheriger jährlicher Kredit von der Hälfte dieses Betrags. Zur Zeit, da Alfred Werner die Leitung des chemischen Unterrichts an der Universität übernahm, war der Institutskredit natürlich etwas größer geworden, nach den Begriffen, 
die wir heute haben, aber immer noch ungenügend. Der Chemikergeneration von heute, die in fürstlich ausgestatteten Laboratorien arbeitet und der fast unbeschränkte Mittel zur Verfügung stehen, fällt es schwer zu glauben, daß noch in den ersten zwei Jahrzehnten unseres Jahrhunderts beim Institutsleiter von seiten der Universitätsverwaltung Reklamationen eingingen, wenn infolge des Neuanschlusses einer oder zweier Wasserstrahlpumpen der Wasserverbrauch des Instituts etwas gestiegen war, oder daß Hochschulkommission und Regierungsrat eine gründliche Untersuchung vornahmen, ob es nicht möglich wäre, statt eines Autoklaven, der 1000 Franken kostete, einen etwas billigeren anzuschaffen.

Der Name Professor Werner führte trotzdem immer größere Scharen von Schülern dem Institut zu. Der Hörsaal, für etwa 130 Mann berechnet, mußte schließlich fast das Doppelte aufnehmen. In den Fensternischen, in den Zwischengängen, rings um den Experimentiertisch herum standen, saßen und drängten sich die Studierenden. Es waren Zustände, die gebieterisch eine Änderung heischten, und Werner hat seinen ganzen Einfluß dafür eingesetzt. Im Jahre 1905 wurde sein sehnlichster Wunsch erfüllt: der Kanton Zürich beschloß den Neubau eines chemischen Universitätsinstitutes, das im Sommer 1909 bezogen werden konnte. Am 17.April 1909 fand in Anwesenheit von Vertretern kantonaler und städtischer Behörden, der Universität, des Polytechnikums und schweizerischer und fremder Universitäten die Einweihung statt. Es sprachen Regierungsrat ERnst, der Rektor Prof. Dr. Kleiner sowie Alfred Werner. Letzterer formte einige Gedanken über die Aufgaben des neuen Instituts. Er sagte, es verfolge zwei Zwecke: Forschung und Unterricht. Als Hochschulinstitut habe es in erster Linie der Forschung zu dienen. Der Unterricht, den er an zweite Stelle rücken möchte, erleide durch die Zuweisung auf den zweiten Platz keinen Schaden, im Gegenteil, er gewinne durch eine erfolgreiche Forschung. Forschung und Unterricht hätten Hand in Hand zu gehen, und auf diese Notwendigkeit sei beim Bau und der Ausstattung des neuen Instituts Rücksicht genommen worden. Die neue Arbeitsstätte, modern ausgebaut und eingerichtet, bot ihrem initiativen Leiter die Möglichkeit, seine großangelegten Untersuchungen unter relativ günstigen äußeren Umständen auszubauen.

Der kosmopolitische Zug, der sich, wie wir hörten, in der ganzen Universität Zürich im vergangenen Jahrhundert bis zum Ersten Weltkrieg geltend machte, war auch im chemischen Institut zu spüren. Fast alle Chemiedozenten waren Ausländer: neben Werner, dem geborenen Elsässer, der zweite Ordinarius, Professor Haruthiun Abeljanz, ein Armenier. Weiterhin Pro- 
fessor P. Pfeiffer, gebürtig aus Elberfeld (Rheinland), PD Dr. Dilthey aus Rheydt (Rheinland), PD Dr. August Bischler aus Karlsruhe, PD Dr. Karl Schall aus Brooklyn bei New York, PD Dr. Adolf Grün aus Wien, PD Dr. JANTsGh aus Österreich, PD Dr. BAudisch, ebenfalls Österreicher, PD Dr. J. V. DuBsky aus Böhmen.

Einzig Dr. Отто Kум, ein am Institut arbeitender Privatgelehrter, stammte aus der Schweiz, aus Zürich. Dieses international zusammengesetzte Dozentengremium, das im großen ganzen kollegial zusammenarbeitete und, geführt von der festen Hand des Chefs, erfreulich gut harmonierte, umgab ein Fluidum, in welchem jede nationalistische Note fehlte, das im Gegenteil von einer weltoffenen Mentalität beherrscht wurde.

Bei der Studentenschaft, den Chemiestudenten, war diese Weltoffenheit und Internationalität vielfach bis zu einem bohémien-artigen Gehaben gesteigert. Wenn, wie wir gehört haben, die Zahl der ausländischen Studierenden an der Universität Zürich in jenen Jahren bis zu $50 \%$ betrug, so war sie unter den Chemiestudenten meistens noch wesentlich größer, in einigen Jahren hob sie sich bis auf $\mathbf{9 0 \%}$. Unter diesen Chemikern gab es sehr viele Russen, Polen, Tschechen, Österreicher, verhältnismäßig viele jüdischer Religion, aber daneben auch deutsche Corps-Studenten, die einen anderen Menschenschlag repräsentierten. Trotzdem kam es zwischen diesen verschieden gearteten Gruppen von Kommilitonen nie zu irgendwelchen größeren Reibereien, im Gegenteil herrschte im ganzen Institut Solidarität und Eintracht. Natürlich waren Fleiß und Leistungen der einzelnen Studenten sehr verschieden, die meisten arbeiteten wohl viel weniger, als dies heute von den Chemiestudenten verlangt wird, und wenn sich aus diesem Grund eine Doktorarbeit über mehr als zwei Jahre, über drei, vier oder sogar fünf Jahre hinzog, so sank der betreffende Mann in den Augen der Kameraden keineswegs, er wurde im Gegenteil als Lebenskünstler gefeiert.

\section{Alfred Werner als Lehrer}

Alfred Werner war ein vorbildlicher Lehrer. Die Klarheit und Logik seines Denkens machten seine Vorlesungen zu genußreichen Stunden. In den ersten Jahren seiner Dozententätigkeit las er eine Vorlesung über organische Chemie; erst von 1902 an wurde ihm von den Behörden auch die Hauptvorlesung über anorganische Experimentalchemie übertragen. Meisterhaft verstand er das Wesentliche eines Themas herauszuarbeiten und komplizierte Probleme einfach und klar darzustellen. Unter der großen Zahl seiner Schüler suchte er sich die besten als Doktoranden aus, und von diesen wurden die begabtesten schließlich seine Assistenten. Bis zu 25 Doktoranden arbeiteten gleichzeitig unter seiner persönlichen Leitung, und es gehört zu seinen erstaunlichen Leistungen, daß er ohne besondere Mühe alle diese Arbeiten gleichzeitig übersehen und lenken konnte. 
Werner besuchte mit großer Regelmäßigkeit täglich zweimal seine Doktorandensäle, einmal vormittags, das zweite Mal nachmittags. Er ging von Doktorand zu Doktorand und wechselte mit jedem ein paar Worte. «Nun?» begann er das Gespräch und meinte damit, was es Neues gäbe; er setzte also voraus, daß in den sechs Stunden, die seit seinem letzten Besuch vergangen waren, vielleicht eine neue chemische Entdeckung gemacht worden sei! Wenn das - wie verständlich - meistens nicht der Fall war, ließ er sich von seinem Doktoranden über dessen Laboratoriumssorgen berichten und versuchte sodann, ihm Ratschläge für neue Versuche zu geben.

Die Arbeitstechnik war bei den Wernerschen Arbeiten verhältnismäßig einfach. Für die Herstellung der zahllosen Metallkomplexsalze, die er durch seine Assistenten und Doktoranden darstellen und untersuchen ließ, brauchte man in der Regel als Laboratoriumsgeräte nur einige Bechergläser, Kristallisierschalen und Trichter: man brachte z.B. zwei geeignete Ausgangsstoffe in einem geeigneten Lösungsmittel - meistens Wasser zusammen und versuchte hierauf, das entstandene Reaktionsprodukt durch Kristallisation zu isolieren. Von der verfeinerten, vielseitigen Laboratoriumstechnik, welche z.B. die organische Chemie heute verwendet (hohe und tiefe Temperaturen, Druck, Katalisatorenwirkung, Chromatographie, Belichtung usw.) war in der Wernerschen Komplexsalzchemie kein Platz. In einem Theaterstück, das die Chemiestudierenden am Chemiker-Weihnachtsabend des Jahres 1911 aufführten, wurde beschrieben, wie ein Wernerscher Doktorand vor seinem Laboratoriumsplatz steht und sich bemüht, aus einer Lösung das gesuchte Kobaltsalz kristallisiert abzuscheiden, die Hauptbeschäftigung, der sich die Doktoranden zu widmen hatten:

\author{
Man reibt und kratzt \\ Und nicht geschwatzt. \\ Man zählt auf drei, \\ Und stöhnt dabei. \\ Kratzt nach der Laus, \\ So fällt es aus. \\ Und neun ist eins, \\ Und zehn ist keins, \\ Das ist das Kobalteinmaleins.
}

Die letzten drei Zeilen lehnen an Goethe an, der Anfang aber gibt ein ungefähres Stimmungsbild aus dem Wernerschen Doktorandenlaboratorium.

Jeder Doktorand hatte die von ihm neu hergestellten Verbindungen selbst zu analysieren, was verhältnismäßig viel von seiner Arbeitszeit be- 
anspruchte und vielleicht ab und zu die Genauigkeit der Analyse ein wenig beeinflußte.

$\mathrm{Da}$ die beiden Hauptvorlesungen in anorganischer Chemie und organischer Chemie, die Werner hielt, nicht nur von Chemikern, sondern auch von anderen Naturwissenschaftern und Medizinstudenten besucht wurden, hatte er Jahr für Jahr eine große Zahl von Prüfungen abzunehmen. RICHARD Willstätter hat in seiner Selbstbiographie Aus meinem Leben Alfred Werner als einen sehr strengen Examinator geschildert (p. 163); er schreibt, daß er seine Doktoranden, die ihre Dissertationen an einer Universität einreichen mußten, da das Eidgenössische Polytechnikum damals noch nicht das Recht besaß, Doktor-Promotionen vorzunehmen, nicht zu Professor Werner ins Doktorexamen schickte, weil «dessen Methode zu examinieren, oft etwas abschreckend war». Und Willstätter fährt fort: «Es war bekannt, daß Werner z. B. eine Stunde lang über Phenanthrenderivate prüfen konnte. Es ist schwer zu verstehen ..., daß ein so bedeutender und erfahrener Universitätslehrer solche Fehler in einer wichtigen Funktion des Hochschulunterrichts machen konnte.» Diese Ausführungen decken sich indessen keineswegs mit den Erfahrungen vieler seiner Schüler. Unter diesen war im Gegenteil bekannt, daß Professor Werner nicht besonders schwierig prüfte und seine Fragen häufig Gebieten entnahm, die unter den Examenskandidaten bekannt waren und auf die man sich daher vorbereiten konnte.

In einer Theaterposse mit dem Titel Drehen und Spalten, welche am Weihnachtsfest der Universitätschemiker 1911 im Casino Hottingen aufgeführt wurde, erzählte einer der studentischen Schauspieler, er hätte geträumt, Professor Werner habe plötzlich neue Examensfragen formuliert und damit die Doktoranden in arge Verlegenheit gebracht:

\footnotetext{
Dann hat der Alte ganz ungeniert

Examensfragen neu eingeführt.

Aus der letzten genugsam bekannten Affaire

Zog er sich seine neue Lehre.

Da war das früher anders gewesen:

Lauter Harnsäure- und Nikotinsynthesen,

Dann Edelsteine - und seltene Erden,

Damit konnte einer Doktor werden.

Die Sache steht nun anders heute,

Jetzt können nur ganz gescheite Leute

In Zürich chemischer Doktor werden.

So ändern die Zeiten auf dieser Erden.
} 
Es mangelte Alfred Werner keineswegs an Humor und Verständnis für Studentenfröhlichkeit. Das trug dazu bei, daß seine Schüler in ihm nicht nur den großen Forscher und Meister verehrten und bewunderten, sondern in ihm auch den Menschen schätzten und liebten, trotz der rauhen Schale, trotz des scheinbar rauhen Äußeren, das ihn umgab und das ein vertrautes Verhältnis oft etwas erschwerte.

Werner war von heiterer Natur und suchte im Freundeskreis Erholung von der angestrengten Arbeit. «Ich brauche die Ablenkung», pflegte er zu sagen. Fast jeden Abend traf er sich im «Pfauen» oder im «Seehof» mit seinen Freunden; ein Jaß mußte oft die Stunden kürzen. Auch das Schachspiel, das er leidenschaftlich liebte, brachte ihm Zerstreuung. Nach Vorträgen in der Chemischen oder Naturforschenden Gesellschaft blieb er gerne mit Kollegen noch eine Stunde zusammen. Erholung suchte er auch während vieler Jahre auf der Jagd, welcher er in seinem Aargauer Revier während der Jagdzeit jeweilen an den Samstagen oblag.

Mit besonderer Freude nahm er jedes Jahr an den Chemikerkommersen teil, die von den Studenten, die im Chemischen Institut arbeiteten, vor Weihnachten veranstaltet wurden. Allen, welche diese Abende einmal mitmachten, sind sie unvergeßlich geblieben. Unter den Klängen eines Tusches betrat Werner schweren Schrittes den dichtbesetzten Kasinosaal, in welchem nachher ein fröhlicher Kommers begann und in manchen Jahren von den Studenten auch Theaterstücke gespielt wurden, die sich natürlich immer um ihn, den «Alten», als Hauptfigur drehten. An diesen Weihnachtskommersen nahmen auch seine ganze Familie, seine Freunde, viele Universitätskollegen bis zum Rektor sowie ehemalige Schüler teil. Jugendlicher Humor lachte einem aus allen Gesichtern entgegen, und Werner fühlte sich unter allen immer als einer der jüngsten. Durch diese Weihnachtsfeste blieb er mit vielen seiner ehemaligen Schüler noch lange Jahre in Kontakt.

Aus allen Weihnachtsfesten der Universitätschemiker ragte wohl dasjenige besonders heraus, das am 15. Dezember 1911 im Casino Hottingen stattfand. Im Laufe jenes Jahres hatte Werner mit seinen Schülern die Spiegelbildisomerie anorganischer Komplexsalze der Typen

$$
\left[\begin{array}{c}
\mathrm{Mex}_{\mathrm{y}}^{\mathrm{en}_{2}} \\
\mathrm{en}
\end{array}\right],\left[\mathrm{Me}_{\mathrm{x}_{2}}^{\mathrm{en}_{2}}\right],\left[\mathrm{Me}(\mathrm{en})_{3}\right]
$$

entdeckt und damit die Richtigkeit seiner Oktaeder-Theorie für solche anorganische Komplexsalze sichergestellt. Dieses besonders wichtige Ergebnis seiner Forschungen bedeutete für Alfred Werner einen Triumph und für seine Schüler einen Grund zu besonderer Freude. Diese allgemeine Befriedi- 
gung und dieser Stolz fanden denn auch am Weihnachtskommers des 15. Dezember 1911 ihren Ausdruck. Die «Spaltung» der Komplexsalze in die nach rechts und nach links drehenden Formen wurde von den Studenten in der Theaterposse Drehen und Spalten verherrlicht, die schließlich damit ausklang, daß alle Studenten und Gäste in den Schlußgesang und dessen Refrain

Ja vom Morgen bis Abend spät

Wird jetzt nur noch gedreht, ja gedreht...

einstimmten und das «Drehen » in einen von alt und jung getanzten Wiener Walzer auslief.

$\mathrm{Ob}$ das Nebeneinanderhergehen von intensiver Forschung und Lehrtätigkeit mit ebenso intensiver Geselligkeit und Fröhlichkeit nicht eine zu schwere Belastung für die Gesundheit Werners war - wer möchte es beurteilen. Das Außerordentliche war ihm angeboren: in der Arbeit, in der Pflichterfüllung und auch im geselligen Leben.

\section{Alfred Werners Verhältnis zu Kollegen}

Eine so eigenwillige, kraftstrotzende, nicht leicht zu Kompromissen bereite Natur, wie sie Alfred Werner eigen war, ist oft keine günstige Grundlage für langewährende Freundschaften und für ein Vertrauensverhältnis mit Kollegen. Trotzdem hatte Werner in dieser Hinsicht nicht allzu große Schwierigkeiten. Mit seinen jüngern Kollegen am Chemischen Institut (Professor Paul Pfeiffer, den Privatdozenten Dr. Grün, Dr. Jantsch, Dr.Baudisch u.a.m.) unterhielt er ein ausgesprochen gutes persönliches Verhältnis, das aber in erster Linie deswegen keine Reibungsflächen aufwies, weil sich diese jüngeren Kollegen ihrem Chef widerspruchslos unterordneten. Während vieler Jahre hatte Professor Werner in Paul Pfeiffer, der seit 1908 als Extraordinarius im Chemischen Institut wirkte, einen wissenschaftlich ausgezeichnet ausgewiesenen und menschlich sehr sympathischen Kollegen, der ihn in allen seinen Intentionen aufs beste unterstützte und ihm half, die Koordinationslehre durch experimentelle Forschung weiter auszubauen und zu stützen. Als Werner 1893 zum Extraordinarius für Chemie an die Universität berufen wurde, wirkte am Chemischen Institut bereits H.Abeljanz als Professor. Dessen Wahl war in einer Zeit erfolgt, als bei der Besetzung von Lehrstühlen bisweilen die politische Einstellung der Kandidaten eine Rolle spielte. Abeljanz war keine Forschernatur, er hat in seinem Leben nur drei oder vier kleinere wissenschaftliche Mitteilungen veröffentlicht, und diese waren auch nicht hochwertig. Trotzdem bekam die von 


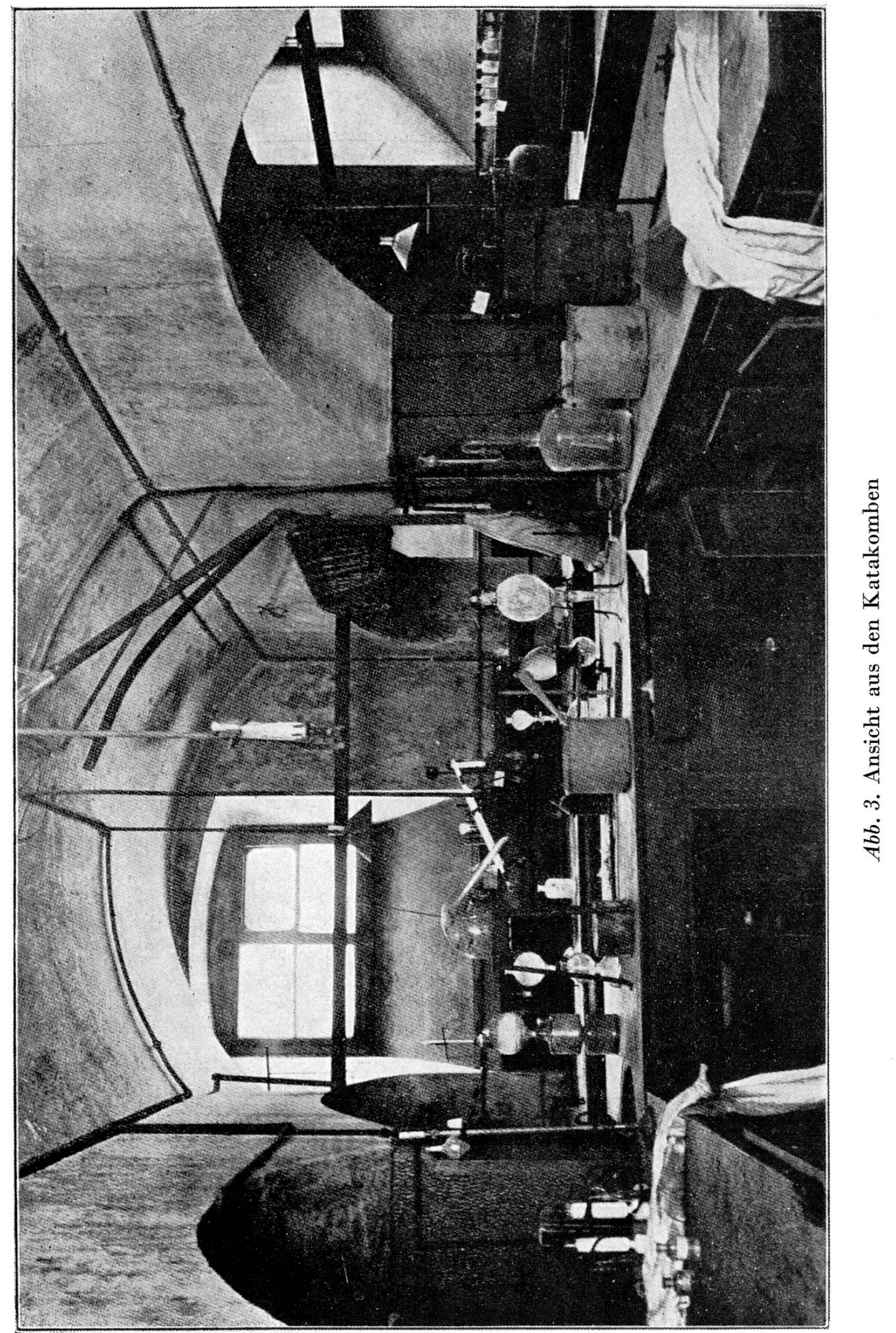




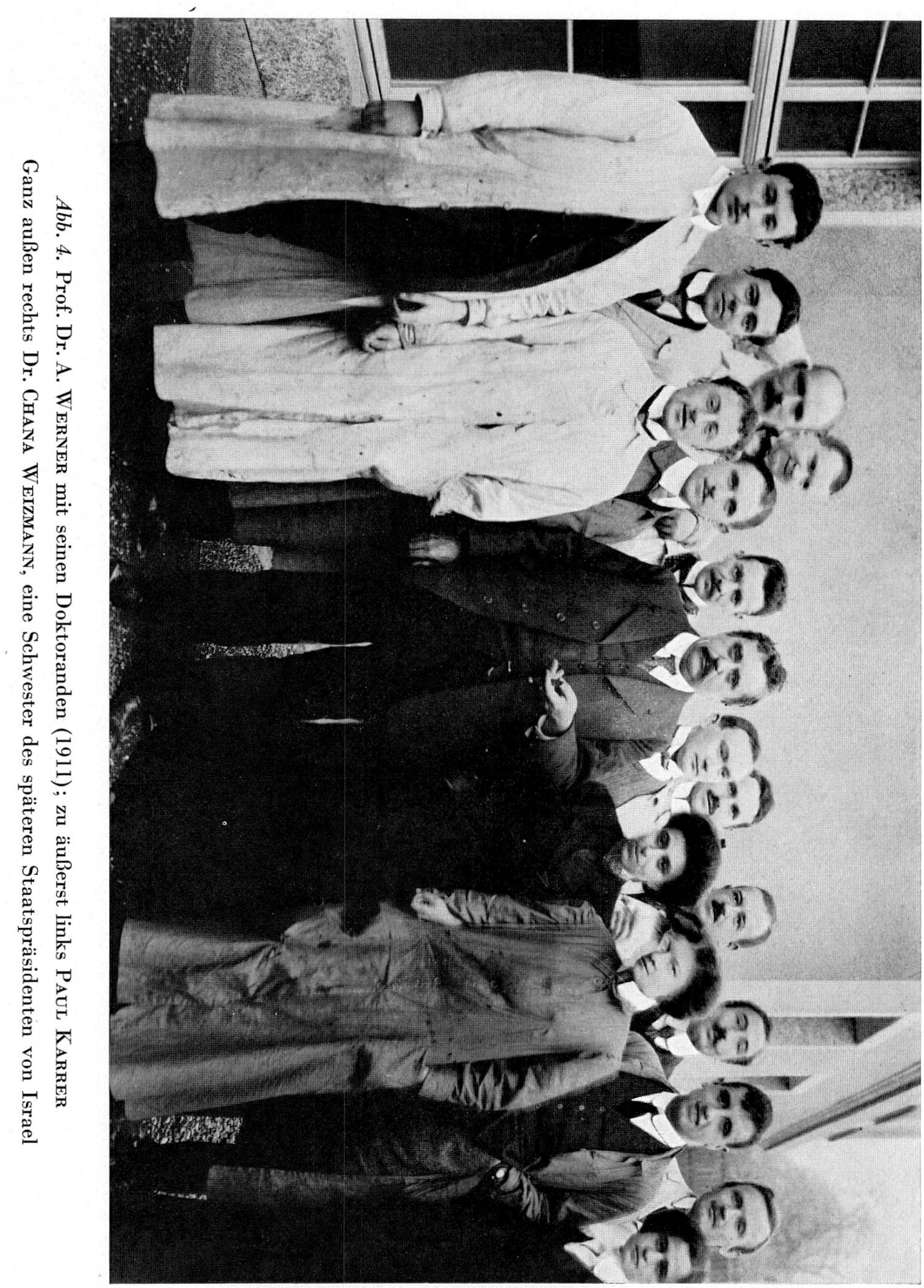


ihm geleitete analytische Abteilung jedes Jahr, wie andere Universitätsinstitute, vom Staat einen, allerdings bescheidenen Forschungskredit. Da er diesen für wissenschaftliche Arbeiten nicht benötigte, machte er, was unter diesen Umständen das Vernünftigste war: er kaufte für den zur Verfügung stehenden Betrag Platingeräte (Schalen, Tiegel, Destillationsgefäße). So kam es, daß dem Chemischen Institut nach dem Rücktritt von Abeljanz eine verhältnismäßig große Menge von Platinutensilien zufiel. $\mathrm{Da} ß$ sich zwischen Werner und Abeljanz unter diesen Umständen kein Vertrauensverhältnis bilden konnte, ist begreiflich. Professor Werner, der nach seinem Eintritt in den Lehrkörper des Chemischen Institutes zunächst nur organische Chemie las, die vor ihm auch sein Vorgänger Victor Merz doziert hatte, wünschte bald, daß ihm Professor Abeljanz auch die Experimentalvorlesung über anorganische Chemie abtrete. Daß Werner als führender Anorganiker dafür ganz besonders qualifiziert war, kann nicht bestritten werden. Die Erziehungsbehörden entsprachen daher seinem Wunsch; aber diese neue Verteilung des Lehrstoffs führte zu einer tiefen Verstimmung der beiden Kollegen.

Richard Willstätter hat in seiner Selbstbiographie Aus meinem Leben über die Art und Weise, wie die Zurückdrängung von Professor Abeljanz angeblich erfolgte, folgendes geschrieben (S. 169): «Das zweite Ordinariat der Universität bekleidete Abeljanz, ein Armenier, der mit Werner selbstverständlich schlecht stand. Werner arbeitete lange Zeit leidenschaftlich daran, seinen Kollegen Abeljanz zu entfernen ... So kam es vor, daß er nach der Vorlesung von Abeljanz in den Hörsaal eindrang und die Tafel mit den nicht eben glücklichen Formulierungen und Gleichungen abphotographierte, um seinen Eingaben an die Regierung überzeugendes Material beizufügen.» Wer diese Behauptung dem Biographen zugetragen hat, läßt sich nicht abklären; sie ist jedenfalls unwahr. Keiner der jüngeren Kollegen und Assistenten Werners, die damals im Chemischen Institut arbeiteten, hat jemals von diesen Photographien gehört; Professor Werner, der eine zwar rauhe, aber durchaus offene Natur besaß, wäre zu einer solchen Handlungsweise nie fähig gewesen. Außerdem existiert ein Schreiben von Professor Werner an die ihm vorgesetzte Behörde, die Erziehungsdirektion, in welchem er rät, Professor Abeljanz im Lehrkörper zu behalten, obwohl die Fakultät anderer Meinung war. Abeljanz leitete von nun an das chemische Laboratorium für Mediziner und hielt Vorlesungen über analytische Chemie.

Mit seinen Kollegen am Eidgenössischen Polytechnikum traf Werner hauptsächlich in der Zürcher Naturforschenden Gesellschaft und insbeson- 
dere in der Zürcher Chemischen Gesellschaft zusammen. Letztere hatte sich aus kleinen Anfängen, in dem $\mathrm{Maß}$ als sich die Chemie an der Universität und der Technischen Hochschule entfaltete, zu einer Vereinigung entwickelt, die auf die Forschung anregend wirkte. Die Sitzungen fanden in einem Bierlokal statt, im ersten Jahrzehnt dieses Jahrhunderts im Restaurant «Pfauen». Die Vortragenden waren die an den beiden Hochschulen wirkenden Dozenten; ausländische Gäste, die heute ihre Rundreisen durch die chemischen Gesellschaften aller Länder ausführen, gab es damals noch wenige. Die Zürcher Chemiedozenten berichteten in den Sitzungen über ihre neuesten Forschungsergebnisse, und an ihre Mitteilungen schlossen sich anregende Diskussionen an. Wie lebhaft das wissenschaftliche Leben in der Gesellschaft war, kann man aus einer Sitzungsprotokollnotiz ersehen, in der es heißt, daß die Versammlung einstimmig beschlossen habe, daß in Zukunft nach zehn Uhr abends keine neuen Vorträge begonnen werden dürfen!

Viele Entdeckungen und Erfindungen, die in der zweiten Hälfte des letzten Jahrhunderts und im ersten Dezennium des 20. Jahrhunderts in Zürich auf dem Gebiet der Chemie gemacht wurden, sind zuerst in der Zürcher Chemischen Gesellschaft einem weiteren Kreis von Fachleuten bekanntgegeben worden. Wislicenus, Victor Meyer, Victor Merz, Arthur Hantzsch, Wilhelm Weith, Eugen Bamberger, Bolley, Emil Kopp, Georg Lunge, Richard Willstätter, Alfred Werner und viele andere sprachen dort und kreuzten ihre Klingen. Der Schreibende erinnert sich z.B. an einen Vortrag Willstätters, in welchem dieser von der ihm mit E. Waser gelungenen Synthese des cyclo-Oktatetraens sprach und aus dessen großer Reaktionsfähigkeit die Schlußfolgerung zog, daß die Thielesche Theorie, welche den reaktionsträgen, aromatischen Charakter des Benzols auf die intramolekulare Absättigung der sechs Partialvalenzen zurückführte, nicht richtig sein kann. Hierauf meldete sich Alfred Werner zum Wort und sagte, er glaube nicht, daß man dies aus den mitgeteilten Versuchsergebnissen schließen könne, da man nicht wisse, ob die Lage der C-Atome im cyclo-Oktatetraen eben ist wie im Benzol oder ob cyclo-Oktatetraen keine ebene Struktur besitzt - womit Werner einen schwachen Punkt der Beweisführung richtig erkannt hatte.

Mit seinem Lehrer Arthur Hantzsch, der bald nach der Wahl Werners zum Professor an die Universität die Zürcher Wirkungsstätte verließ und nach Würzburg übersiedelte (1893), verband Werner auch in späteren Jahren tiefe Freundschaft. Ebenso bestanden zwischen ihm und Professor 
Nölting in Mülhausen, den er, wie wir hörten, seit seinen Jugendjahren kannte, herzliche Beziehungen. Mit Willstätter unterhielt Werner in den Jahren, in denen letzterer in Zürich lebte (1905-1912) ebenfalls persönlichen Kontakt. Daß dieser nicht sehr tief reichte, sondern an der Oberfläche blieb, war bei der großen Verschiedenheit der beiden Charaktere nicht erstaunlich. Wenn es für die Äußerlichkeit dieser Beziehungen noch eines Beweises bedurft hätte, so wurde dieser von Willstätter selbst dadurch erbracht, daß er in seiner Autobiographie über Werner verschiedene Anekdoten erzählt, die alle Werner in einem ungünstigen Licht erscheinen lassen -, aber auch alle die tröstliche Eigenschaft haben, frei erdichtet zu sein.

Unter den engeren Fakultätskollegen waren es insbesondere die Professoren Arnold Lang und Alfred Kleiner, mit denen Werner eng befreundet war und mit denen er sich oft beim Billardtisch traf. Als Lang als erster durch den Tod abberufen wurde, hat Werner sich noch mit Kleiner allein am Billardtisch eingefunden, bis ihm auch dieser Freund durch den Tod entrissen wurde. In späteren Jahren unterhielt Werner auch mit dem Botaniker Professor Hans Schinz freundschaftlichen Verkehr. Professor Lang, der eine Berufung als Nachfolger HAEGKels nach Jena ausgeschlagen hatte, und Werner, der Berufungen nach Wien und Würzburg ablehnte, haben sich unter sich bisweilen durch die Anrede «Hofrat» und «Geheimrat» geneckt, Titel, welche die beiden überzeugten Demokraten mit einem schmunzelnden Lächeln quittierten.

Da Werner durch seine Koordinationslehre in der anorganischen Chemie und verwandten Gebieten revolutionierend wirkte, mußte er notgedrungen älteren Auffassungen von Kollegen, die über analoge Probleme arbeiteten, entgegentreten. Er hat dies immer mit großem Takt getan und Polemiken, wenn immer möglich, vermieden. Sein am stärksten in Erscheinung tretender wissenschaftlicher Gegner war S.M. JörgenseN in Kopenhagen, dessen Lebenswerk auch auf dem Gebiet der anorganischen Komplexsalze lag, von denen er viele entdeckt und näher untersucht, aber, von alten Vorstellungen eingenommen, bezüglich ihrer Konstitution unrichtig aufgefaßt hat. Diesen Jörgensenschen Arbeiten mußte Werner daher öfters entgegentreten. Er versuchte diese sachlichen Differenzen nicht zu persönlichen Zwistigkeiten sich entwickeln zu lassen. Als er nach Entgegennahme des Nobelpreises von Stockholm nach Zürich zurückreiste, wollte er Professor Jörgensen in Kopenhagen eine Besuch machèn, doch kam dieser wegen schwerer Erkrankung von Jörgensen leider nicht zustande. 


\section{Krankheit und Tod}

Die letzten Jahre seines Lebens sind Werner zu einer harten Prüfung und großen Qual geworden. Auf dem Höhepunkt seines Lebens, kurz nachdem ihm die Mitwelt als Ausdruck ihrer Bewunderung und Dankbarkeit für seine wissenschaftlichen Leistungen den Nobelpreis verliehen hatte, erkrankte er (1915) an einem schweren Leiden. Die Ärzte, welche den Ernst der Krankheit erkannten, drangen auf Aufgabe der Arbeit.

Es war die Zeit des Ersten Weltkrieges. Dieser hatte auch für das Chemische Institut der Universität Zürich tiefgreifende Folgen. Die zahlreichen ausländischen Studenten, besonders Russen und Polen, schwärmten wie ein Bienenschwarm aus und versuchten, in ihre Heimat zurückzukehren. Die wenigen Chemiestudenten schweizerischer Nationalität aber mußten großenteils zu ihren militärischen Einheiten einrücken. Die Hörsäle und Laboratorien leerten sich; im Hause herrschte eine gedrückte Stimmung. Diese wurde noch dadurch erhöht, daß Professor P. Pfeiffer eine Berufung an die Universität Rostock erhielt, und er die Absicht bekundete, ihr Folge zu leisten. Als der Schreibende im Frühjahr 1918 als a.o. Professor in das Institut eintrat, waren in diesem nur noch fünf Doktoranden (und Doktorandinnen) tätig.

Es war für Werner ein schwerer Entschluß, seine Lehr- und Forschertätigkeit aufzugeben, die seinem Leben Inhalt und Zweck gewesen waren. Zweimal versuchte er noch, seinen Urlaub unterbrechend, die Tätigkeit im Institut wieder aufzunehmen. Aber die Krankheit war stärker als seine Willenskraft. Nach langem, schwerem Kampf, der ihm qualvolles, seelisches Leid brachte, mußte er sich schließlich ergeben. Am 15. November 1919 schloß er die Augen.

Werner ist auf dem Gipfel seines Ruhmes vom Tod ereilt worden. Bis in die letzte Zeit seiner wissenschaftlichen Tätigkeit war er mit neuen Problemen beschäftigt, und man hätte von seinem Geist und klaren Verstand wohl noch manche neue Schöpfung erwarten können. Trotzdem ist sein Lebenswerk nicht unvollendet. Als abgerundetes Ganzes steht es vor unseren Augen. Die Koordinationslehre hat Werner der Nachwelt als eine gesicherte monumentale Schöpfung hinterlassen. Was er nicht mehr erlebte, aber vielleicht ahnte, ist die große Bedeutung, zu welcher diese Lehre auch in anderen Wissenschaften später gelangte, in der Kristallographie, Mineralogie, Physik, aber auch in gewissen Gebieten der organischen Chemie. 
Man wird vielleicht die Frage aufwerfen, warum ein Mann vom Format und der Bedeutung Alfred Werners nicht eine größere Schule hinterlassen hat: tatsächlich haben nur wenige seiner Schüler die Dozentenlaufbahn eingeschlagen (Professor P. Pfeiffer, Professor P. Karker, Professor G. Jantsch, Professor O. Baudisch, PD Dr. Grün). Dies ist wohl auf zwei Gründe zurückzuführen: einmal auf die Tatsache, daß der größte Teil seiner Schüler Ausländer waren, die nach erfolgter Promotion wieder in ihre (meistens östliche) Heimat zurückkehrten, und zum anderen darauf, daß der akademische Beruf in jenen Jahrzehnten noch als eine sehr unsichere und brotlose Kunst, ja als ein Wagnis galt, wenn der junge Dozent finanziell nicht völlig unabhängig war.

Die Mitwelt hat Werner die Anerkennung für seine ungewöhnlichen Leistungen nicht versagt. Mehrere ehrenvolle Berufungen wurden ihm zuteil: 1899 eine solche an die Universität Wien, 1902 eine an die Universität Basel, 1905 an die Eidgenössische Technische Hochschule, 1910 eine Berufung nach Würzburg - er hat sie alle aus Anhänglichkeit an die Universität Zürich ausgeschlagen. Die Universität Genf und die Eidgenössische Technische Hochschule verliehen ihm Ehrendoktorate. Eine große Zahl inländischer und ausländischer gelehrter Gesellschaften wählten ihn zum Ehrenmitglied bzw. korrespondierenden Mitglied; es sind dies die Königliche Gesellschaft der Wissenschaften in Göttingen, die Physikalisch-medizinische Societät von Erlangen, die Société de Physique et d'Histoire Naturelle de Genève, die Société Impériale des Amis d'Histoire Naturelle, d'Anthropologie et d'Ethnographie Moskau, der Physikalische Verein Frankfurt a. M., die deutsche Bunsengesellschaft, die Société Vaudoise des Sciences Naturelles Lausanne, die Chemical Society London, die Academy of Natural Science of Philadelphia, die American Chemical Society Washington. Die Société Chimique de France verlieh Werner die Leblanc-Médaille. Er wurde zum Officier de l'instruction publique en France ernannt. 1913 wurde ihm die höchste Ehrung, der Nobelpreis für Chemie, zuerkannt. Die Schweizerische Chemische Gesellschaft ließ es sich nicht nehmen, diese hohe Auszeichnung in einer Sondertagung am 2. Mai 1914 in Neuenburg zu feiern, an welcher Professor Fr. Fichter aus Basel in seiner Festrede einen Überblick über die wissenschaftlichen Arbeiten des Gefeierten gab. Gleichzeitig wurde ihm eine von Bildhauer Vibert geschaffene «Werner-Plakette» überreicht und die Gründung eines «Werner-Fonds» bekanntgegeben, aus welchem für hervorragende wissenschaftliche Arbeiten junger Kollegen ein «Werner-Preis» verliehen werden kann. 


\section{Die neue Valenz- und Koordinationslehre}

Es wurde schon weiter vorne erwähnt, daß am Beginn der wissenschaftlichen Entwicklung A. Werners vor allem drei bedeutende Veröffentlichungen stehen: seine 1899 der Zürcher Universität eingereichte Dissertation Über die räumliche Anordnung der Atome in stickstoffhaltigen Molekülen; ferner der erste Teil seiner für die Habilitation am Eidgenössischen Polytechnikum bestimmten Abhandlung Beiträge zur Theorie der Affinität und Valenz (1892) und schließlich die große Veröffentlichung Beiträge zur Konstitution anorganischer Verbindungen.

Die erste dieser drei Abhandlungen erschien unter den Namen Arthur Hantzsch und Alfred Werner in den Berichten der Deutschen Chemischen Gesellschaft. Sie ist aber nach der Bezeugung von Hantzsch das geistige Eigentum seines Schülers Werner, indem er in einer Anmerkung sagt: «Schließlich erfüllt der Unterzeichnete nur eine Pflicht der Gerechtigkeit, indem er für den Fall, daß obigen Entwicklungen einige Bedeutung zuerkannt werden sollte, die Erklärung abgibt, daß diese gemeinsam mit Hrn. A. Werner veröffentlichte Theorie in allem Wesentlichen das geistige Eigentum des Hrn. Werner ist ...»

In intuitiver Weise gab Werner in dieser Arbeit neue Vorstellungen über die Konfiguration der Oxime und verwandter stickstoff haltiger Verbindungen. Er machte die Annahme, daß die drei Valenzen des Stickstoffs unter Umständen aus der Ebene heraustreten und in den Ecken eines Tetraeders, in dessen vierter Ecke der Stickstoff selber steht, zur Wirkung kommen. So wurde es möglich, die Stereoisomerie der Oxime nicht auf eine Raumisomerie in bezug auf den Kohlenstoff, sondern in bezug auf den Stickstoff zu deuten. Werner wurde damit zum Begründer der Stereochemie des Stickstoffs. Seine Auffassung erfuhr durch alle weiteren Untersuchungen Bestätigung und ließ sich ohne weiteres auf verwandte Körperklassen (Alkylbenzhydroximsäuren u.a.) übertragen.

Die zweite der erwähnten drei Abhandlungen veröffentlichte Werner 1891 in der Vierteljahrsschrift der Zürcher Naturforschenden Gesellschaft; da keine verbreitete chemische Fachzeitschrift ein Referat über diese Arbeit brachte, wurde sie zunächst in Fachkreisen wenig bekannt. Auszüge aus ihr fanden später Aufnahme in den beiden Lehrbüchern, die Werner 1904. und 1905 erscheinen ließ (Lehrbuch der Stereochemie und Neuere Anschauungen auf dem Gebiet der anorganischen Chemie). Die genannte Abhandlung beschäftigt sich mit der Theorie der Affinität und Valenz. Im Gegensatz zu 
KeKulÉ begründete Werner die Auffassung, die Valenz sei nicht eine gerichtete, in ihrer Stärke fixierte Einzelkraft, sondern eine Energiegröße, welche, an der Oberfläche der kugelig gedachten Atome wirkend, in verschiedener Weise aufspaltbar sei, so daß sie sich in stärkere und schwächere, in mehr oder in weniger viele Valenzeinheiten zerlegen läßt. Diese neue Anschauung wurde zuerst auf die Affinitätsverhältnisse beim Kohlenstoff angewandt. Eine neue Erklärung der Benzolformel, des unterschiedlichen Verhaltens der ortho-, meta- und para-Stellungen, eine neue Interpretation organischer Konfigurationsformeln und eine Erklärung stereochemischer Umlagerungen waren Früchte dieser Arbeit.

Schon zwei Jahre später, 1893, erschien die dritte und wichtigste der vorerwähnten Abhandlungen unter dem Titel: Beiträge zur Konstitution anorganischer Verbindungen, in welcher Werner seine Ansichten über die Valenz in die Koordinationslehre weiter entwickelte. Diese Koordinationslehre postuliert, daß sich in anorganischen Molekeln die verschiedenartigsten Atome und Atomgruppen um ein als Zentrum («Koordinationszentrum») wirkendes Atom (am häufigsten Metallatom) räumlich anordnen. Die Zahl der Atomgruppen, die um das Zentralatom Platz finden, ist die Koordinationszahl. Die koordinativ gebundenen Atomgruppen haben keinen Ionencharakter, sie stehen in «innerer» oder «erster» Sphäre und sind fest mit dem Koordinationszentrum verkettet; außerhalb der «ersten» Sphäre finden die ionogenen Reste ihren Platz.

Über diese Arbeit erschien in Band 26, Seite 351 (1893) der Berichte der Deutschen Chemischen Gesellschaft ein Referat, das die darin enthaltenen Gedanken kurz und trefflich wiedergibt und welches zeigt, was die neue Lehre bei den anorganischen Verbindungen zu leisten vermochte. Sie zeigt den jungen Gelehrten bereits auf der Höhe seiner Erfindungs- und Gestaltungsgabe. Durch die experimentellen Arbeiten, die er mit seinen Assistenten und Schülern in den folgenden zwanzig Jahren ausführte, mußte an der ursprünglichen Form der Koordinationslehre nichts Wesentliches geändert oder korrigiert werden, indem sie sich bis in die Einzelheiten bewährte. Alles Folgende war nur Ausbau der genialen Intuition.

Es wird immer etwas Unerklärliches bleiben, wie ein Gelehrter, ohne lange Vorstudien, ohne große experimentelle Erfahrung, bisweilen ohne eigene experimentelle Arbeit, plötzlich zu einer neuen Erkenntnis gelangen kann, die auf einem großen Gebiet der Wissenschaft umwälzend wirkt und früher unerklärliche Dinge schlagartig klärt. Wie sich diese Gedankenarbeit vollzieht, bleibt dem betreffenden Forscher vielleicht selbst verborgen. 
Werner soll sich wiederholt dahin geäußert haben, daß die Grundgedanken der Koordinationslehre im Anschluß an einen geselligen, fröhlichen Abend bei ihm entstanden seien. Sicheres wissen wir darüber nicht.

Zur weltweiten Verbreitung der Koordinationslehre hat Alfred Werners hervorragende Monographie Neuere Anschauungen auf dem Gebiete der anorganischen Chemie, deren erste Auflage 1905 erschien und die noch drei weitere Auflagen erlebte, viel beigetragen. In dieser hat er eine umfassende Darstellung des gesamten damals bekannten experimentellen Materials gegeben, das die Stütze seiner Koordinationslehre bildete. Dieses Werk ist die Bibel der Chemie der Komplexsalze geworden. Es legt erneut davon Zeugnis $a b$, mit welcher Klarheit Werner einen großen, komplexen Stoff zu gestalten vermochte.

Ganz gegen Ende seiner wissenschaftlichen Forschertätigkeit, im Jahr 1913, hat Werner anläßlich der Entgegennahme des Nobelpreises die Gelegenheit nochmals, diesmal zurückschauend, wahrgenommen, über die Koordinationslehre zusammenfassend zu sprechen. In seinem Nobelvortrag gab er nochmals Rechenschaft über die Entstehung, den Inhalt und die Leistungen dieser Lehre, welche nach 20 jähriger Bewährung immer noch ebenso groß dastand wie im Jahr ihrer Entstehung.

Der Autor dieser «Erinnerungen» hat vor 46 Jahren in einem Nekrolog ${ }^{1}$ und nun nochmals in dem vorliegenden Aufsatz Professor Werner so geschildert, wie er ihn gekannt und während mehreren Jahren gemeinsamer Arbeit erlebt hat. Das Werk Werners war nicht nur reich an wissenschaftlichen Entdeckungen und U̇berraschungen, er hat sich auch anderen Lebensfreuden nicht versagt.

Diese markante Persönlichkeit ist heute bereits von zahlreichen Anekdoten umrankt und umwoben, von denen leider manche ungenau, andere übertrieben und wieder andere sogar unrichtig sind und das Bild des großen Gelehrten und des im Grunde unkomplizierten Menschen Alfred Werner leider oft etwas entstellen.

Zürich, Spyristeig 30

${ }^{1}$ Helv. Chim. Acta 3 (1920) 196. 